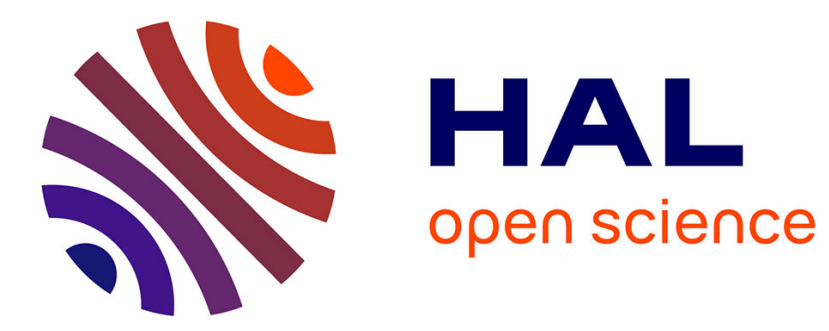

\title{
Lane detection and scene interpretation by particle filter in airport areas
}

\author{
Claire Meymandi-Nejad, Salwa El Kaddaoui, Michel Devy, Ariane Herbulot
}

\section{To cite this version:}

Claire Meymandi-Nejad, Salwa El Kaddaoui, Michel Devy, Ariane Herbulot. Lane detection and scene interpretation by particle filter in airport areas. 14th International Conference on Computer Vision Theory and Applications (VISAPP 2019), Feb 2019, Prague, Czech Republic. hal-02180537

\section{HAL Id: hal-02180537 https://hal.laas.fr/hal-02180537}

Submitted on 11 Jul 2019

HAL is a multi-disciplinary open access archive for the deposit and dissemination of scientific research documents, whether they are published or not. The documents may come from teaching and research institutions in France or abroad, or from public or private research centers.
L'archive ouverte pluridisciplinaire HAL, est destinée au dépôt et à la diffusion de documents scientifiques de niveau recherche, publiés ou non, émanant des établissements d'enseignement et de recherche français ou étrangers, des laboratoires publics ou privés. 


\title{
Lane detection and scene interpretation by particle filter in airport areas
}

\author{
Claire Meymandi-Nejad ${ }^{1,2}$, Salwa El Kaddaoui ${ }^{1}$, Michel Devy ${ }^{1}$, Ariane Herbulot ${ }^{1,3}$ \\ ${ }^{1}$ CNRS, LAAS, Toulouse, France \\ ${ }^{2}$ INSA de Toulouse, Toulouse, France \\ ${ }^{3}$ Univ. de Toulouse, UPS, LAAS, F-31400 Toulouse, France \\ \{cmeymand, selkada,devy, herbulot $\} @ l a a s . f r$
}

Keywords: lane detection, inverse perspective mapping, particle filter, polygonal approximation, aeronautics

\begin{abstract}
Lane detection has been widely studied in the literature. However, it is most of the time applied to the automotive field, either for Advanced Driver-Assistance Systems (ADAS) or autonomous driving. Few works concern aeronautics, i.e. pilot assistance for taxiway navigation in airports. Now aircraft manufacturers are interested by new functionalities proposed to pilots in future cockpits, or even for autonomous navigation of aircrafts in airports. In this paper, we propose a scene interpretation module using the detection of lines and beacons from images acquired from the camera mounted in the vertical fin. Lane detection is based on particle filtering and polygonal approximation, performed on a top view computed from a transformation of the original image. For now, this algorithm is tested on simulated images created by a product of the OKTAL-SE company.
\end{abstract}

\section{INTRODUCTION}

A lot of studies have been conducted in the automotive domain on computer vision, in order to perform scene interpretation required to provide reliable inputs for automatic driving or driver assistance, etc. As such, the detection of road surface markings has been thought of as a key factor for road and lane tracking from a vehicle. Lane detection and road analysis are currently used in driver assistance systems and provide multiple advantages. Lane detection is the main element used for road modelling, intelligent driving, vehicule localization or obstacle detection.

Two main types of methods are commonly used for lane detection: model-based and feature-based methods. Model-Based methods, often defined for vehicule friendly roads, either on highways or on urban streets, are based on strong models: camera parameters, width and curvature of the road, position of the vehicule with regards to the scene elements (Asif et al., 2007), (Deng et al., 2013), (Loose and Franke, 2010), number of road lines (Chapuis et al., 1995), etc. Prior knowledge is necessary to build the road model as they allow to predict where road surface markings could be in images, but it must be updated with measurements extracted from these images. These models present better robustness but require more computational resources and strong assumptions on the scene geometry. Feature based- methods use a combination of low level features such as color models, contrast, shape, orientation of groups of pixels and so on. They are of lower computational complexity compared to model-based methods, which is an advantage when dealing with real-time systems (Sun et al., 2006), (Lipski et al., 2008), (Hota et al., 2009). However, these techniques may fail in case of shadowing or occlusions which is prompt to happen when dealing with obstacle detection.

Less studies have been devoted to scene interpretation in aeronautics, when considering aircraft navigation on taxiways (Theuma and Mangion, 2015), (Tomas et al., 2017). Road models differ from airport ones (gates, taxiway, runway) which also are complex and varying environments requiring a frequent update of their models. This paper proposes a scene interpretation method based on weak models exploited to detect taxiway horizontal markings and beacons. We use a combination of observations and prior knowledge of the scene to approximate the complex functions describing the ground line shapes with a set of pre-selected samples (Sehestedt et al., 2007), (Jiang et al., 2010).

Assuming the airport is a local 2D environment, a top view image is first computed by an Inverse Perspective Mapping (IPM) transformation, applied to raw images acquired from a camera mounted in the aircraft vertical fin. Different observation maps are then created from this top view, and used by a par- 


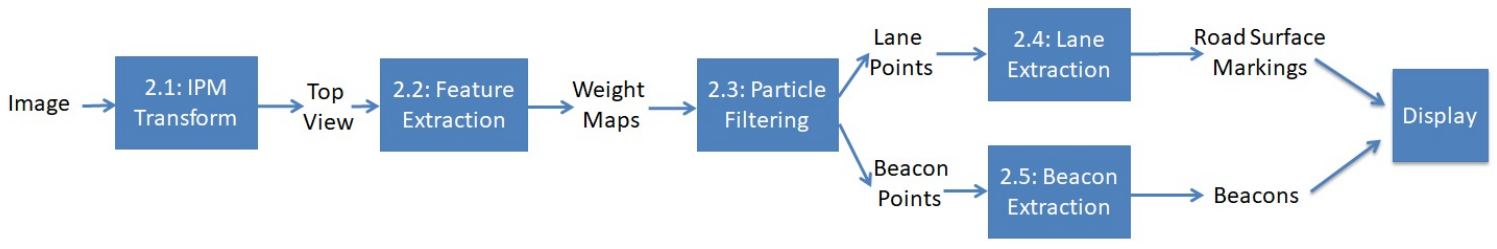

Figure 1: Functions for interpretation of airport scenes

ticle filter for the detection of lines. The use of the particle filter is motivated by the need to implement a probabilistic method instead of a deterministic one, as we work on weak models. Clusters of points are collected from the particle filter, arranged and merged to determine the line equations by polygonal approximation. Other elements such as beacons are detected on the taxyway in the same way, and added to the detected lines to produce an augmented reality view of the taxiway.

The next section describes different functions integrated in our scene interpretation module. Results are presented in the section 3, while contributions and current works are summarized in the section 4.

\section{METHODS}

The different functions described in this section are shown on Figure 1: they currently aim at building an augmented view to be displayed in the cockpit; the scene representation will be used later for alerting the pilot if a risky situation is detected, or for autonomous navigation of the aircraft on taxiways.

\subsection{Inverse Perspective Mapping (IPM)}

As presented in (Deng et al., 2013), the scene can be presented, in the Euclidian space, either in the world coordinate system or in any chosen image coordinate system. We decided to use a top view transformation by applying an Inverse Perspective Mapping to our frame. The coordinate systems used are illustrated in Figure 2.



Figure 2: Representation of the camera coordinate system (blue) and IPM coordinate system (red)

Providing that we define a point in the world coordinate system (respectively in the image coordinate system) by the following coordinates $P_{W}\left(X_{W}, Y_{W}, Z_{W}\right)$ (respectively $P_{C}\left(X_{C}, Y_{C}, Z_{C}\right)$ ), we will note any transformation in the world to camera coordinate system process $P_{W / C}\left(X_{W / C}, Y_{W / C}, Z_{W / C}\right)$. The expression of a point in the camera coordinate system is expressed in Equation 1.

$$
P_{C}=\left[\begin{array}{lll}
R_{1} & R_{2} & R_{3}
\end{array}\right] \cdot P_{W}+t
$$

$R_{1}, R_{2}$ and $R_{3}$ represent the rotation between the two coordinate systems in the three dimensions while $t$ is the translation part of the movement. $P_{C}$ can also be defined as in Equation 2

$$
P_{C}=\left[X_{W / C} Y_{W / C} Z_{W / C}\right] \cdot P_{W}+0_{W / C}
$$

The homography $H$ needed to change from the world coordinate system to the image coordinate system can be found in Equation 3. As we project the image on the ground to create a top view, the $Z_{W / C}$ is not taken into account. The homography used in our algorithm can be found in Equation 4, where $K$ is the intrinsic matrix of the camera, $D$ is the height of the camera in relation to the ground and $\theta$ correspond to the tilt value of the camera.

$$
\begin{gathered}
H=K \cdot\left[X_{W / C} Y_{W / C} 0_{W / C}\right] \\
H=K \cdot\left[\begin{array}{ccc}
-1 & 0 & x_{1 n} \\
0 & \sin (\theta) & D \cdot \cos (\theta) \\
0 & -\cos (\theta) & D \cdot \sin (\theta)
\end{array}\right]
\end{gathered}
$$

To compute an IPM image adapted to our specifications, we perform a discretization of the image where the dimensions of one cell are defined as $(\delta X, \delta Y)$. This permits to describe the coordinate $(i, j)$ of each pixel in the IPM image in relation to its $(X, Y)$ coordinates in the image coordinate system. Equation 5 links the $(i, j)$ and $(X, Y)$ coordinates, where $D_{i}$ and $D_{j}$ are the size of the IPM frame. The intensity of the $(i, j)$ cell is interpolated from the value of the $(u, v)$ cell in the image. The link between the $(i, j)$ and $(u, v)$ coordinate is despicted in Equation 6. The Figure 3 exposes the IPM process. Results of the IPM are presented in Figure 4. All the following image treatments will be applied to the resulting image shown in Figure 4(b).

$$
\left[\begin{array}{l}
i \\
j \\
1
\end{array}\right]=\left[\begin{array}{ccc}
0 & 1 / \Delta Y & D_{i} \\
-1 / \Delta X & 0 & D_{j} / 2 \\
0 & 0 & 1
\end{array}\right] \cdot\left[\begin{array}{c}
X \\
Y \\
1
\end{array}\right]
$$




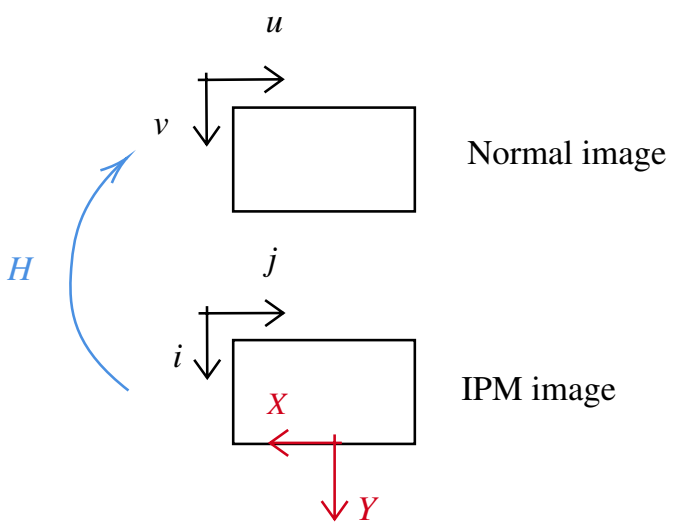

Figure 3: Creation of IPM frame from camera frame

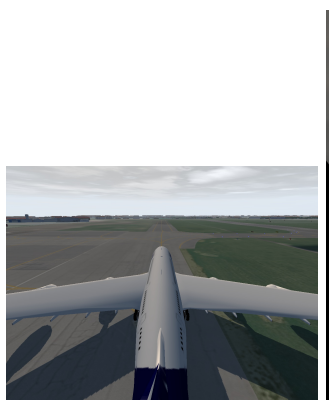

(a)

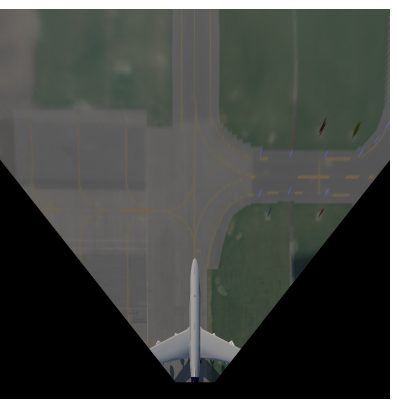

(b)
Figure 4: (a) Original image, (b) IPM transformation

$$
\text { s. }\left[\begin{array}{l}
u \\
v \\
1
\end{array}\right]=H \cdot\left[\begin{array}{l}
X \\
Y \\
1
\end{array}\right]
$$

\subsection{Weighted Maps Computation}

The transformed IPM image is used to create several maps. The goal of these maps is to compute a weight for each pixel before launching a particle filter on the IPM image, for lane detection. The weight of a pixel will increase as it is closer to the reference color of the object to detect and its belonging to a detected border of the object.

\subsubsection{Edge map computation}

Our algorithm uses two types of edge maps. One is used to detect any border in the image whereas the other is mainly based on the lane analysis. Combined, they offer varied information.

The first map is created by using the Sobel gradient on the image intensity. The IPM image obtained in Figure 4(b) is converted into a grayscale image and a Sobel gradient is performed on the created image. An exponential treatment is then applied to the result values in order to increase the contrast between

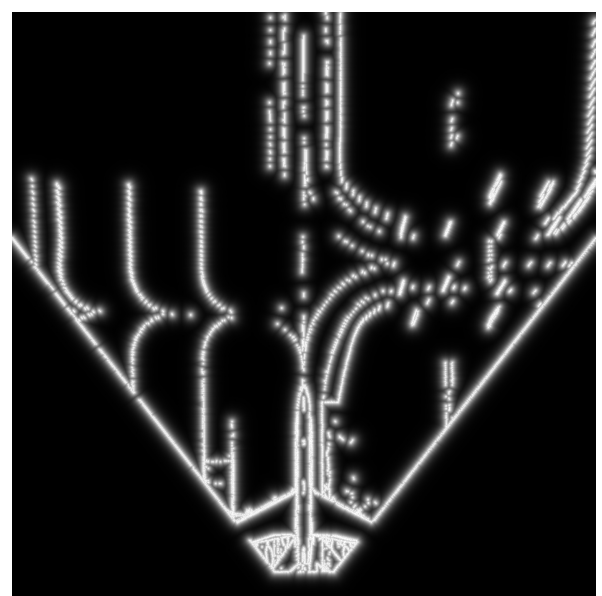

Figure 5: Edge map - Sobel Gradient

black and white pixels. Figure 5 is an example of the provided map where the blurry effect is created by our exponentional treatment. The exponential parameters are chosen with regards to our data to express the noise in the measurement and confidence in the measurement. The second edge map is based on the model of a lane and a method proposed by (Bertozzi and Broggi, 1998). In the aviation field, specifications are numerous and common to all airports. The sizes of the lanes that can be found in an airport are regulated. We use the knowledge of the lane size for this edge detection. The IPM frame is first converted in the HSV (Hue, Saturation, Value) color-space, where we select the saturation channel. The yellow color of the lanes becomes much more expressive in this representation. Based on the knowledge of the road, our algorithm skims the resulting image of the saturation channel in order to create a binary version of the image. Each pixel is compared to its left and right neighbors, in terms of saturation value and a threshold is fixed to discriminate the pixels belonging to a lane from the others. Once this binarization is computed, we apply a distance transform, based on the algorithm described by (Felzenszwalb and Huttenlocher, 2012) and implemented in OpenCV, to the image obtained. The result of this treatement is shown in Figure 6.

The map created thanks to the Sobel gradient enables our algorithm to detect geographical informations such as borders between the tarmac and the grass or beacons. It can help to create a model of the scene and filter false positive detections. The second map is focused on the known size of the main lane to follow in an airport and reduce the number of borders to examine. To detect only a lane, we will use the second map whereas the beacons and tarmac delimitations are computed with the first map.

Using the Sobel Gradient map combined with the 


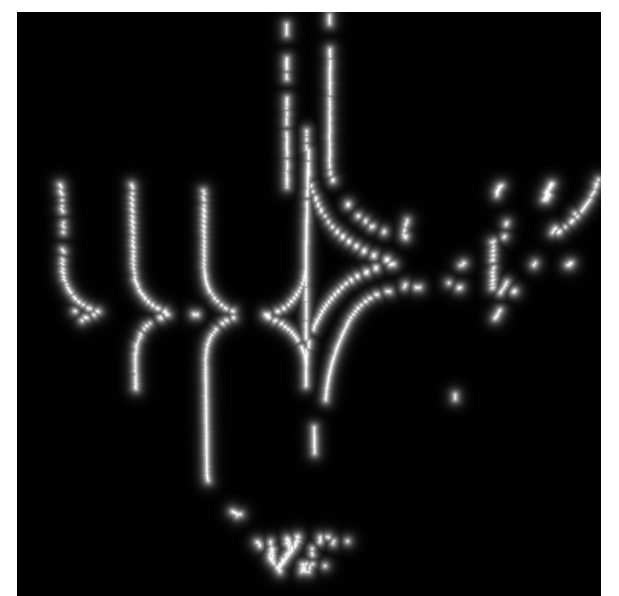

Figure 6: Edge map - Lane model

second map could be efficient for double checking as the size of the lane can vary with partial occlusion or curvy parts but the combination of the two maps increase the computation time. We know that when the lane becomes curvy, its apparent size in the IPM image can be reduced and the lane might not be detected by our algorithm. This is why we apply treatments to the second map, to add noise in order to simulate our confidence in the measurement and increase the detection rate.

\subsubsection{Color map computation}

The color of the various lanes that can be encountered are regulated and defined in specific range of yellow and white. We can use these specifications in our model, to create a map of similarity between the color of a pixel and the reference color. To ensure that the algorithm is more robust to wheather phenomenons such as shading, we compute the color map in the LAB color-space. A patch of reference color is defined in the LAB color-space and our algorithm applies a convolution between this patch of the reference color and the color IPM image (see Figure 4(b)) in order to determine a distance between the two patches of pixels, based on the A and B channels. Figure 7 shows the resulting color map.

\subsubsection{Global maps computation}

We combine the Edge and Color maps defined above to create a measure map. A value is attributed to each pixel and represents the multiplication of a color weight by the edge weight, computed thanks to the two previous maps. A specific exponential treatment is applied to the color and edge weights to increase the contrast between a pixel belonging to a lane and the others. We also create a binarized version of this map

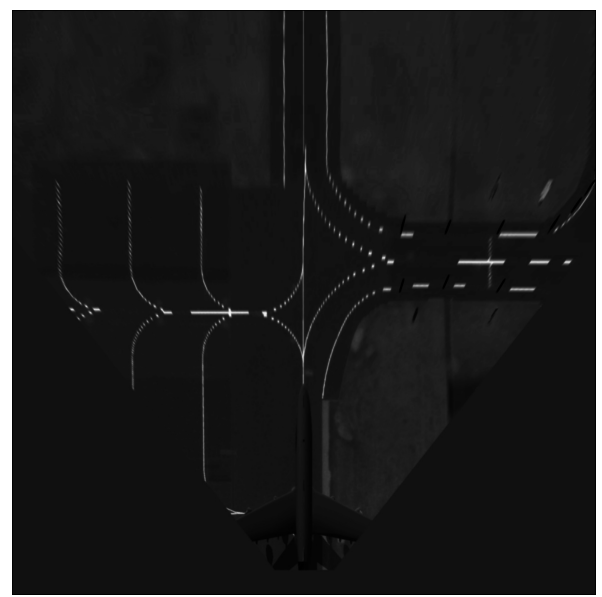

Figure 7: Color map

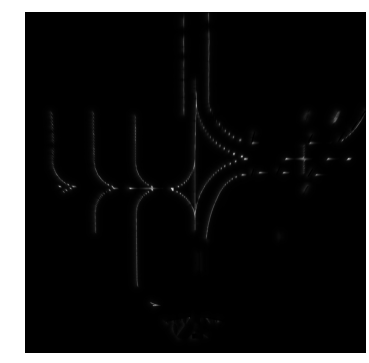

(a)

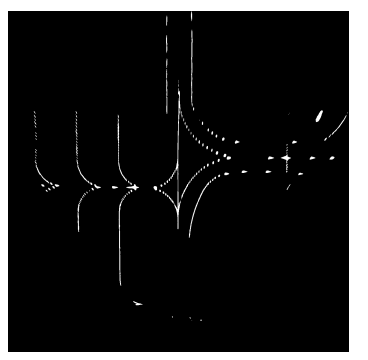

(b)
Figure 8: (a) Weights map, (b) Binarized map

used for the particle initialization while the weights will be used for the particle survival estimation. The results are presented in Figure 8, where it can be seen that most of the additional noise brought by the edge map is filtered by the use of a combination of the two maps, which increase the accuracy of the particle filter and reduce the computation time.

\subsection{Particle Filter}

\subsubsection{Particle Initialization}

As explained in the introduction, we decided to use a particle filter for the lane detection, using the bootstrap filter or Sequential Importance Resampling (SIR). The Figure 9 represents its operation. We will note the state variable $x_{t}$ a random variable describing the state of a system at time $t, y_{t}$ a random variable describing the sensor measurements at time $\mathrm{t}, w_{t}$ a variable describing the computed correction of the predictions (importance weight) and $q\left(x_{k} \mid x_{0: t-1}, y_{0: t}\right)$ the proposal distribution. The particle filter approximates the probable distribution of $X_{t}$ with a set of samples, or particles noted $p\left(x_{0: t} \mid y_{1: t}\right)$ computed with $\left\{x_{0: t}^{(i)}, w_{0: t}^{(i)}, i=1 . . n\right\}$. Particles are updated through a 


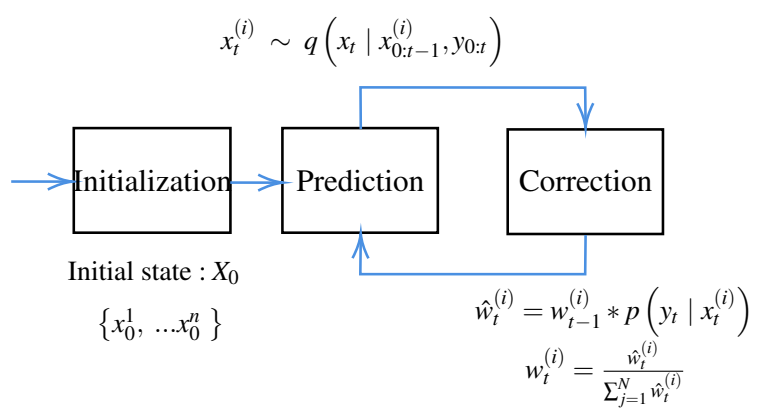

Figure 9: Principle of the particle filter

series of predictions based on the prior knowledge of the system and corrections of these predictions based on the sensor measurements. The estimation of the current state variable $\hat{x}_{t}$ is selected with the argmax function.

The initialization is done by scanning the global map row by row, from bottom to top, until white pixels are detected, these pixels represent the probable starting point of a lane. The first line composed of white pixels will be used as the initialization. Any group of connected white pixels is defined as a segment on which we randomly scatter a defined number of particles.

\subsubsection{Particle correction and future prediction}

The particle filter implemented in our algorithm scans the image every $n$ rows, where $n$ has been defined to be small enough to detect possible new starts of lanes and big enough to reduce the computational time. At initialization, these particles are associated to a weight given by the global map. The weights are used at time $t>t_{0}$ to give higher importance to most probable particles, thus resulting in better prediction for future particles through multinomial resampling. The weights of the different particles are normalized and create a cumulative weights scale. Random values between 0 and 1 are computed and represented in this scale where they are matched to specific particles.

To predict new particules, we only use, as past positions, the positions of the particles related to the random values. The new particles will follow a Gaussian distribution centered around the past particle.

\subsubsection{Cluster mergings and separations}

As we said before, the airport areas are difficult to model because they can easily vary. While the particle filter scans the image row after row, new lanes can appear, old lanes can disappear and several lanes can merge to form only one remaining lane. It is also possible that false positives trigger new lanes detections.
The initialization of particle clusters for new detected lanes at time $t$ and the prediction of future particles for already existing clusters are done before launching the $t+1$ step. To manage the cases where a lane can disappear or when a false positive has created a suspicion of lane, we implemented several thresholds. A survival likelihood is computed for each particle, with regards to its weight and the weights of the other particles, such as in Equation 7 where $w_{i}$ is the normalized weight, $n$ is the number of particles and $p_{i}$ is the survival likelihood. Once several particles have reached a determined value of survival likelihood, the cluster of particles is terminated.

$$
p_{i}= \begin{cases}1, & \text { if } w_{i} \geq \frac{1}{n} \\ n * w_{i}, & \text { otherwise }\end{cases}
$$

In case of merging of lanes or separation of a detected lane in multiple lanes, we decided to study the barycenter of each cluster of particles. Based on their proximity, our algorithm either merges the clusters in one or creates new clusters for new detected lanes. An illustration of this cases can be found in Figure 10 where the green pixels represent particles with high survival likelihood while red pixels represent particles with low survival likelihood. It also shows as new lanes are tracked and clusters are merged.

\subsection{Line extraction}

\subsubsection{Polygonal approximation}

Once the particle filter processing of the image is finished, the algorithm output is a list of all the created clusters and the positions of its particles at each iteration of the particle filter. We select the most prominent particle for each iteration based on the particle weight. We then choose to approximate the detected lanes by affine equations. As the clusters can sometime include curves, we perform a polygonal approximation. This increases the number of clusters but the approximated lanes for the cluster positions are more accurate. As the lanes in the tarmac will not be too curvy, we can use this method without increasing the computational time.

\subsubsection{Line fitting and cluster merging}

Most of the time, we can see that a unique lane can be separated in multiple clusters by the previous steps. It also happens when there is a crossing in the image, where a straight lane will separate in two and reappear few meters ahead. As we need to give useful information to the pilots, we decided to recreate this lanes by merging the clusters. Figure 11 is an example of two 


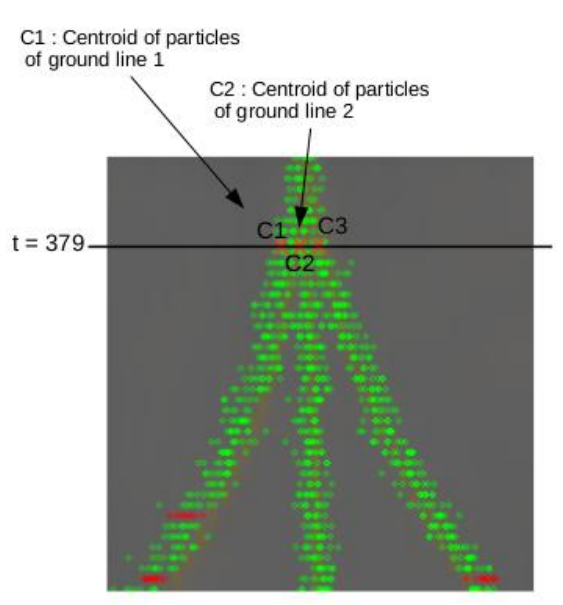

(a)

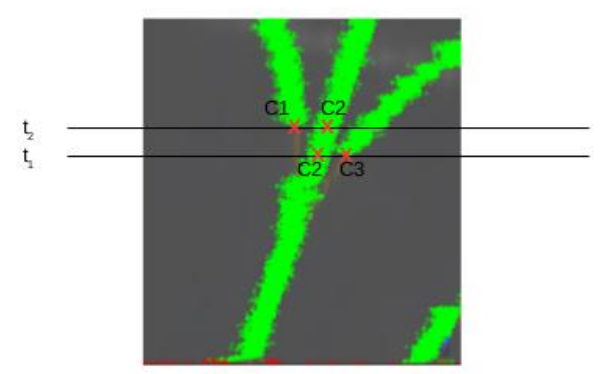

(b)

Figure 10: (a) Fusion of clusters, (b) New lane tracking

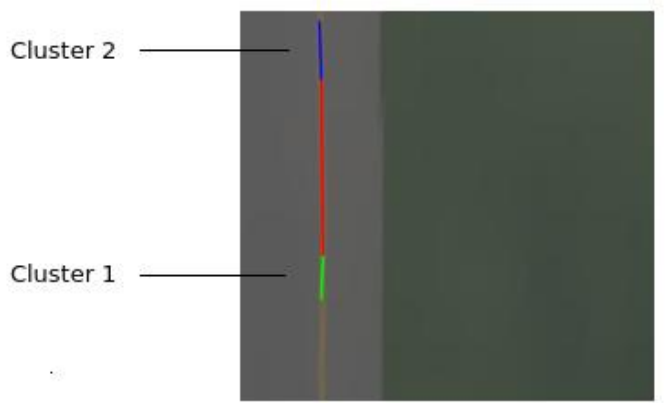

Figure 11: Clusters to be merged together

clusters to be merged together to form one lane. For each cluster of positions, we use a line fitting algorithm. Lines can either be vertical or horizontal. To be able to compute the cluster line equation, we differenciate these two cases. Once lines have been computed for each cluster, we compare them between each other in order to merge similar clusters and recreate the observed lanes in the image.

\subsection{Beacon extraction}

The main goal of our algorithm is to perform scene analysis and lane detection. In the sections 2.2 and 2.3, we defined the method for the lane detection but it can also be applied for beacon detection or tarmac limits detection. For the lane detection, we used an edge map based on the model of a vertical straight lane. In order to detect the horizontal lanes, we perform a transposition on the image before launching our algorithm again. The results of the two iterations are then mixed together. The process is also similar for beacons detection, for which we use the Sobel edge map and a specific color map. As we use the IPM view, the beacons are deformed and can be seen as little blue stripes. Those are detected by our algorithm, from which we select the first position, as it represents the real position of the beacon.

\section{RESULTS}

In order to illustrate the results of our algorithm, we simulated a case of line intersection, as we can see in Figure 4(b), which is a case that can commonly occur in airport areas.

The airplane model used for this simulation is a A380 and the camera, which resolution is 1280pX960p and horizontal field of view is 80 degrees, is mounted on its fin at a distance D of 19,5 meters above the ground and an angle $\theta$ of 20 degrees. Our goal is to perform scene analysis to improve pilots comprehension of the environment. Our specifications is to analyse the scene up to 350 meters ahead. For our algorithms, the image is represented by an IPM view of resolution 1400pX1400p where each pixel represents an area of $25 \mathrm{~cm} X 25 \mathrm{~cm}$.

Figure 12 gives an overall view of the algorithm results. In Figure 12(a) the green pixels represent the estimation of the beacon position. Figure 12(b) shows the results of the particle filter on the vertical lines, with red points representing the particles with low survival likelihood, where lines estimations are highlighted in green in Figure 12(c). Figure 12(d) and 12(e) follow the same process but on the transposed IPM image.

To produce an image which could be easily used by pilots, we return to the original view by performing an inverse homography to highlight the detected lanes and beacons in the real image. The result is shown in Figure 13, where Figure 13(a) shows the combination of the clusters detected in Figure 12(b) and 12(d).

The estimation of the lines positions is quite accurate. The positions of the beacons, colored in red 


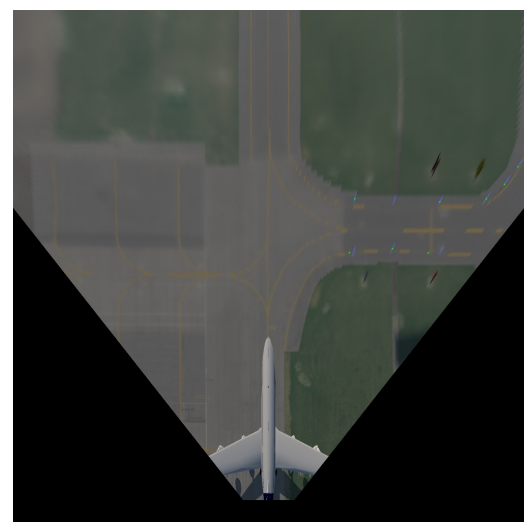

(a)

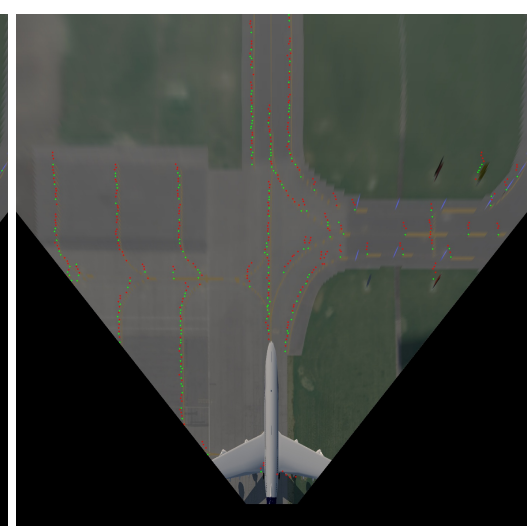

(b)

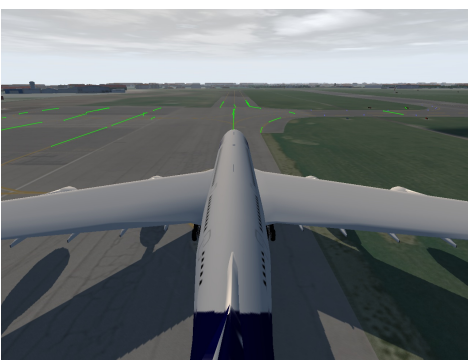

(c)

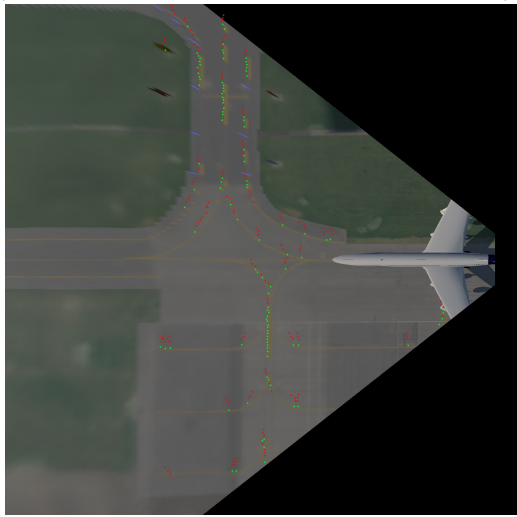

(d)



(e)

Figure 12: (a) Detected beacons based on the original image, (b) Position of clusters from the particle filter based on the original image, (c) Detected lines based on the original image, (d) Position of clusters from the particle filter based on the transposed image, (e) Detected lines based on the transposed image

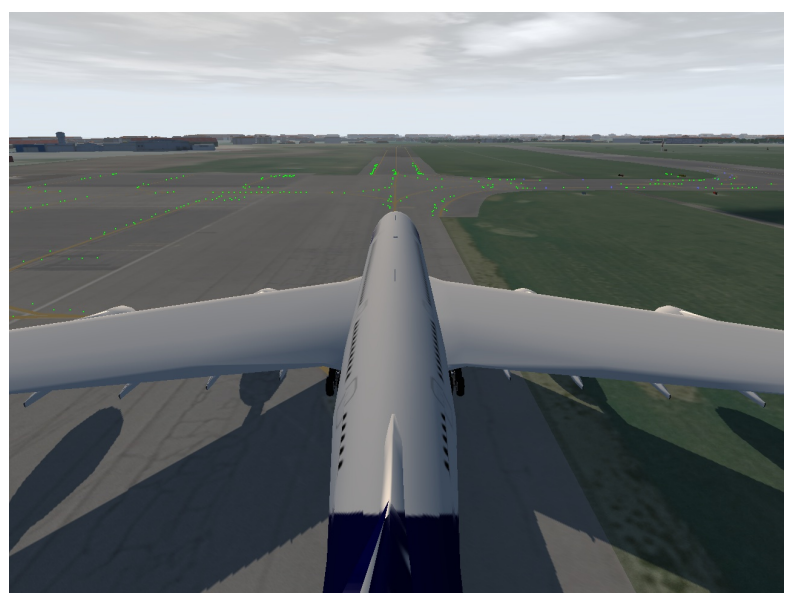

(a)

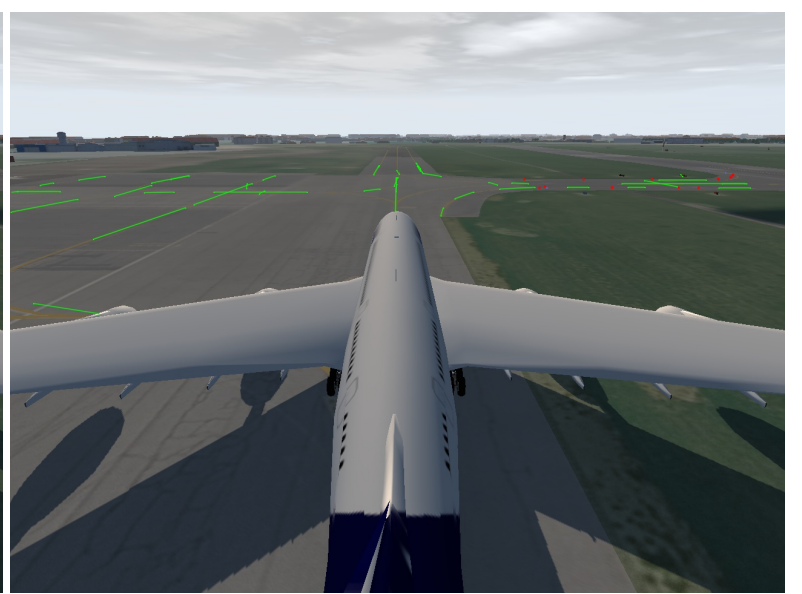

(b)

Figure 13: (a) Result of the combined position of clusters, (b) Result of the combined lines (green) and beacons (red)

in Figure 13(b) are nearly all matching the original beacon positions. However, our algorithm does not precisely detect the curve part of the taxiway lanes. Any curved part brings noise to the lane detection be- cause our lane detection algorithm is based on an edge map established by the lane model. On curved parts, the lane model is modified and not accurately represented in the resulting edge map. Merging the two 
edge maps in one with the same operation used to create the global weights map could improve our algorithm accuracy on curved parts without significantly increasing the computation time.

\section{CONCLUSIONS AND PERSPECTIVES}

In this paper, we presented an accurate algorithm for scene analysis and lane detection in an airport taxiway, based on an IPM transformation and a particle filter. For the moment, the algorithm is based on a straight lane model. For curved parts, as the lane model changes, we will need to explore new models, in order to detect any type of lanes in the scene and give a more detailed view to the pilots. Extra tuning of the road size threshold based on the modelling of the size decrease in curved parts would greatly increase the lane detection.

By now, images used for our algorithm validation are produced by a simulator. They are modelised at daytime, with clear weather conditions. Our objective is to generalize our algorithm to other weather conditions such as night time, rainy or foggy days. To achieve this, we plan to increase our dataset by combining information from RGB camera and IR camera. As the simulation tool can be tuned to match our camera, we can work on images similar to real ones.

Our future goal is to use the combination of lanes and beacons, with the help of other scene elements such as the tarmac limits and panels on the side of the tarmac for example, to implement a line tracking algorithm. This algorithm will enable us to perform egomotion estimation. This information can then be used in an object detection algorithm also based on an IPM transformation, which goal is to detect objects combining the egomotion estimation and images at time $t$ and $t-1$.

For now, our algorithm is not optimized and is launched on a basic computer, with a computation time of few seconds for lines, beacons and tarmac detection for one image. In the future, we plan to optimize the code and implement it on a dedicated architecture including multi-cores CPU, GPU and FPGA.

\section{ACKNOWLEDGEMENTS}

We would like to thank the OKTAL-SE company for providing a simulation tool which provides images, close to real images, for testing the method.

\section{REFERENCES}

Asif, M., Arshad, M. R., Zia, M. Y. I., and Yahya, A. I. (2007). An implementation of active contour and kalman filter for road tracking. International Journal of Applied Mathematics, 37(2):71-77.

Bertozzi, M. and Broggi, A. (1998). Gold: a parallel realtime stereo vision system for generic obstacle and lane detection. IEEE transactions on image processing : a publication of the IEEE Signal Processing Society, 7(1):62-81.

Chapuis, R., Potelle, A., Brame, J., and Chausse, F. (1995). Real-time vehicle trajectory supervision on the highway. The International Journal of Robotics Research, 14(6):531-542.

Deng, J., Kim, J., Sin, H., and Han, Y. (2013). Fast lane detection based on the b-spline fitting. International Journal of Research in Engineering and Technology, 2(4):134137.

Felzenszwalb, P. F. and Huttenlocher, D. P. (2012). Distance transforms of sampled functions. Theory of Computing, 8:415-428.

Hota, R. N., Syed, S., B, S., and Radhakrishna, P. (2009). A simple and efficient lane detection using clustering and weighted regression. In 15th International Conference on Management of Data, COMAD.

Jiang, R., Klette, R., Vaudrey, T., and Wang, S. (2010). Lane detection and tracking using a new lane model and distance transform. Machine Vision and Applications, 22:721-737.

Lipski, C., Scholz, B., Berger, K., Linz, C., Stich, T., and Magnor, M. (2008). A fast and robust approach to lane marking detection and lane tracking. 2008 IEEE Southwest Symposium on Image Analysis and Interpretation, pages $57-60$.

Loose, H. and Franke, U. (2010). B-spline-based road model for $3 \mathrm{~d}$ lane recognition. 13th International IEEE Conference on Intelligent Transportation Systems, pages 91-98.

Sehestedt, S., Kodagoda, S., Alempijevic, A., and Dissanayake, G. (2007). Robust lane detection in urban environments. 2007 IEEE/RSJ Int. Conference on Intelligent Robots and Systems, pages 123-128.

Sun, T.-Y., Tsai, S.-J., and Chan, V. (2006). Hsi color model based lane-marking detection. 2006 IEEE Intelligent Transportation Systems Conference, pages 1168-1172.

Theuma, K. and Mangion, D. Z. (2015). An image processing algorithm for ground navigation of aircraft. In Bordeneuve-Guibé, J., Drouin, A., and Roos, C., editors, Advances in Aerospace Guidance, Navigation and Control, pages 381-399, Cham. Springer International Publishing.

Tomas, B., Ondrej, K., and Ondrej, P. (2017). Methods and systems for providing taxiway stop bar information to an aircrew. 